\title{
DIAGNÓSTICO DA PRODUÇÃO INTELECTUAL DA PÓS-GRADUAÇÃO EM CIÊNCIA DA INFORMAÇÃO DA UFSCAR NO MOMENTO DE SUA IMPLEMENTAÇÃO: DESAFIOS E POTENCIALIDADES
}

\section{DIAGNOSIS OF THE INTELLECTUAL PRODUCTION OF UFSCAR'S POST-GRADUATION IN INFORMATION SCIENCE AT THE MOMENT OF ITS IMPLEMENTATION: CHALLENGES AND POTENTIALITIES}

\author{
Vanessa Dionello ${ }^{a}$ \\ Luciana de Souza Gracioso ${ }^{b}$ \\ Roniberto Morato do Amaralc \\ Marcela Bassoli ${ }^{d}$
}

\begin{abstract}
RESUMO
Introdução: A qualificação, criação e ampliação de Programas de Pós-Graduação strictu sensu (PPG) são ações estratégicas e fundamentais à produção científica e intelectual brasileira. Consequentemente, estes programas precisam ser constantemente avaliados visando a sua melhoria. No campo da Ciência da Informação (Cl) estas ações têm se intensificado. Objetivo: Elaborar e aplicar uma sistemática para o diagnóstico do potencial de atuação de um PPGCl recém implementado, visando identificar seu comportamento e vocação para a produção cientifica na área. Metodologia: Análise de conteúdo de relatórios do sistema de avaliação da CAPES; identificação e mapeamento da produção cientifica de docentes permanentes do PPGCI da Universidade Federal de São Carlos (UFSCar); estabelecimento de base comparativa da produção intelectual do PPGCI UFSCar, com o PPGCI da Universidade Federal de Minas Gerais. Resultados: A partir da análise dos dados e das comparações estabelecidas, foi possível constatar o engajamento e a pró-atividade do corpo docente do PPGCI UFSCar na submissão dos resultados de suas pesquisas para publicação em eventos e periódicos qualificados na área. Ao mesmo tempo evidenciam-se lacunas na
\end{abstract}

a Graduada em Biblioteconomia e Ciência da Informação pela Universidade Federal de São Carlos (UFSCar). E-mail: vanessa.dionello@gmail.com

b Docente do Departamento de Ciência da Informação da Universidade Federal de São Carlos (UFSCar).E-mail:luciana@ufscar.br

c Docente do Departamento de Ciência da Informação da Universidade Federal de São Carlos (UFSCar). E-mail: roniberto@ufscar.br

d Mestre em Ciência, Tecnologia e Sociedade pela Universidade Federal de São Carlos (UFSCar). E-mail: marcelabassoli@gmail.com 
colaboração entre os pesquisadores, tanto internos ao Programa quanto vinculados a outras instituições. Conclusões: Explicita-se a necessidade de elaborar estratégias visando mobilizar os atores deste processo a investirem na produção científica e em redes de colaboração, de modo que possam contribuir na promoção do impacto científico e social do PPGCl. A metodologia utilizada também se mostrou eficaz para ser usada por outros PPGs em fases iniciais de implementação, que almejem estabelecer estratégias para sua consolidação e crescimento.

Descritores: Ciência da Informação. Produtividade científica. Programa de PósGraduação em Ciência da Informação.

\section{INTRODUÇÃO}

Os desafios inerentes à organização da informação, desvinculados em um primeiro momento de sua dependência tecnológica e computacional, datam de séculos. Desde a Biblioteca de Ebla na Síria ( $3^{\circ}$ milênio a.C.), passando pela constituição de disciplinas e áreas científicas como a Bibliografia, a Bibliologia, a Documentação e a própria Biblioteconomia, a informação (que nem sempre foi denominada assim), o seu registro, a sua representação e a promoção de seu acesso foram investigados. E é na busca da consolidação de um campo de pesquisa e investigação sobre a produção, o comportamento e os usos da informação que a Ciência da Informação $(\mathrm{Cl})$ tem sua origem. Mais pontualmente, em meados de 1960, estudiosos de diferentes áreas do conhecimento e de diferentes países passam a problematizar e a sistematizar os desafios que vinham se apresentando na imbricação do desenvolvimento tecnológico e da produção e acesso a novos conteúdos informacionais, intensificados no pós-guerra.

Na década de 1960 ainda, intensificam-se os estudos métricos, que se aproximam com mais exatidão ao universo da informação científica. Araújo (2018) destaca que o Institute for Scientific Information (ISI) criado por Garfield, tem como objetivo analisar citações bibliográficas de artigos científicos. Esses estudos já seriam uma sofisticação das leis identificadas por Alfred Lotka (produtividade de cientistas, 1926), por Samuel Bradford (dispersão do conhecimento científico, 1934) e por George Zipf (frequência de palavras no texto, 1949). É também nesta década que é apresentada a talvez mais citada 
definição de Ciência da Informação, feita por Borko (1968). Sua definição concentrou, em alguma medida, o entendimento geral sobre esta nova área, no período, e da qual destacamos o seguinte trecho:

A Ciência da Informação está preocupada com o corpo de
conhecimentos relacionados à origem, coleção, organização,
armazenamento, recuperação, interpretação, transmissão,
transformação e utilização da informação" (BORKO, 1968, p. 1,
tradução nossa).

Esta visão de Borko é ampliada, complementada e rebatida por diferentes pensadores no período, mas é justo reconhecer que, mesmo respeitando as limitações conceituais desta tentativa de definição, alguns elementos que caracterizam, em parte, a Cl, podem ser assumidos. Araújo (2006) é um dos autores que sugere uma superação epistemológica para o campo, incorporando a complexidade das ciências humanas e sociais no escopo das discussões sobre a informação. E concordamos com ele.

Mas é no cenário de uma $\mathrm{Cl}$ mais positivista que os estudos métricos passam a ser intensificados, em diferentes áreas do conhecimento, e enquanto instrumento de mensuração da produção bibliográfica. Atribui-se a Pritchard, em 1969, a criação o termo bibliometria, enquanto disciplina voltada ao desenvolvimento e aplicação destas métricas. Mas desde a constituição da Documentação, pelo belga Paul Otlet, que a bibliometria já estava sendo desenhada enquanto técnica para as suas análises bibliológicas. (ARAÚJO, 2006). Assim, longe de se apresentar o percurso gerativo desta disciplina, fazse esta menção a esta sua fase embrionária contextualizando-a no bojo do desenvolvimento do próprio campo da $\mathrm{Cl}$, respeitando evidentemente tantas outras disciplinas que também se circunscreveram neste período para a construção deste campo.

Concomitantemente aos movimentos de criação da $\mathrm{Cl}$ em âmbito global, no Brasil intensificavam-se a criação de novos cursos de bacharelado em Biblioteconomia e Documentação, e já na década de 1970 são criados os primeiros Programas de Pós-Graduação em Ciência da informação (PPGCl) e as primeiras revistas científicas da área. O desenvolvimento da Pós-Graduação em Cl no país se deve principalmente a necessidade de ampliar e especializar o desenvolvimento de produtos e serviços de informação, agora mediados por 
tecnologias de comunicação, que vinham sendo incorporadas em diferentes instâncias no país sobre Ciência e Tecnologia, em especial, no ambiente científico. Assim, as atividades do então IBBD (Instituto Brasileiro de Bibliografia e Documentação, 1954-1975) passam a ser desenvolvidas pelo IBICT (Instituto Brasileiro de Informação em Ciência da Tecnologia), que deu origem ao primeiro PPGCI do Brasil. Coube assim, a este Instituto promover e monitorar as atividades de informação em Ciência e Tecnologia no país.

Frente a esta breve contextualização histórica que situa a $\mathrm{Cl}$ enquanto área voltada a pesquisa de C\&T, e que localiza a criação pioneira da pósgraduação brasileira em $\mathrm{Cl}$, neste mesmo cenário, é que se propõe investigar o movimento de produção científica de um PPGCl, recém-criado, objetivando reconhecer seu comportamento e sua vocação, ou não, para se configurar enquanto Programa produtor de conhecimento para esta área.

Assim, em razão da criação do PPGCI da Universidade Federal de São Carlos (UFSCar), localizada no estado de São Paulo, em junho de 2016, identificou-se a necessidade de se mapear, descrever e caracterizar a produtividade científica dos docentes permanentes vinculados a este novo PPG, com o intuito de estabelecer um primeiro diagnóstico quantitativo, que pudesse subsidiar orientações e estratégias, visando mobilizar os atores deste processo (os docentes permanentes) a investirem em determinadas ações de produção científica. Ações mais racionais e legítimas, que de fato possam promover impacto científico e social e assim, consolidar o PPGCl em construção, enquanto produtor de conhecimento científico para a $\mathrm{Cl}$. De modo pontual, a pergunta que norteou o desenvolvimento deste artigo foi: "o que" e "o quanto" é produzido cientificamente pelos docentes permanentes credenciados no PPGCI UFSCar e o quanto desta produção está condizente com o comportamento da produção científica do campo?

Tendo em vista a criação desse novo programa (PPGCI UFSCar), somado às diversas discussões que envolvem o campo de avaliação da pósgraduação brasileira, foi objetivo deste artigo elaborar e aplicar uma sistemática para o diagnóstico do potencial de atuação de um PPG da área de Ciência da Informação, sob a perspectiva da Avaliação da CAPES (Coordenação de 
Aperfeiçoamento de Pessoal de Nível Superior).

A CAPES é o órgão responsável pela pós-graduação brasileira, e estabelece diretrizes e critérios de avaliação específicos para cada área do conhecimento, de modo que sejam claras as expectativas para um desempenho de qualidade de um PPG. Esses critérios são variados, e o de maior relevância nessa avaliação envolve a análise quanti e qualitativa das produções científicas desenvolvidas nesses programas e que englobam, com diferentes pesos, as teses e dissertações defendidas, livros publicados, trabalhos em anais de eventos e artigos publicados em periódicos científicos. Como constatado por Giuliani et al. (2016, p. 244) é importante

[...] para qualquer programa de pós-graduação stricto sensu atentar para os critérios de avaliação da CAPES, no sentido de ajustar-se e aprimorar-se para que possa conquistar conceitos mais elevados e, assim, oferecer aprendizagem de alta qualidade.

Metodologicamente, este artigo utilizou da análise de conteúdo (BARDIN, 2011) para estabelecer em nível introdutório, uma base comparativa entre a produção científica do corpo docente do PPGCI UFSCar, ativo entre 2016-2017, frente às produções científicas de docentes ativos de um PPGCl de uma Instituição Federal, tal como a UFSCar, e considerado, na ocasião do desenvolvimento deste artigo, de excelência: o PPGCI da Universidade Federal de Minas Gerais (UFMG). Esta base de comparação foi aferida enquanto recurso para projeção e prospecção ao direcionamento das políticas e estratégias de produção e comunicação dos resultados de pesquisa pelo PPGCI UFSCar, recém-inaugurado. Cabe a ressalva metodológica que no momento da coleta de dados, o Programa analisado como base de comparação apresentava uma estrutura que foi remodelada e alterada posteriormente após a avaliação trienal da CAPES publicada em 2017.

Ainda na perspectiva metodológica deste artigo, se mostrou necessário o desenvolvimento de uma análise dos critérios aplicados pela CAPES na avaliação dos PPGCI, a partir da análise dos relatórios de avaliação disponíveis publicamente pela instituição. Especificamente, foi feita uma análise detalhada do documento de área das Ciências Sociais Aplicadas I (CSAI), que passou a 
ser denominada Comunicação e Informação em 2016 . É importante salientar que estes critérios de avaliação são constantemente revistos pelos respectivos representantes de área via grupos de trabalho e que há um empenho significativo destes representantes na consolidação de critérios e indicadores que sejam coerentes e justos ao cenário da pós-graduação brasileira. Em síntese, para o desenvolvimento do diagnóstico proposto, o principal processo de trabalho, envolveu a identificação e a sistematização da produção, intelectual, dos 10 docentes permanentes vinculados ao PPGCI UFSCar, no momento de sua criação. Este diagnóstico inicial procurou indicar em qual perspectiva este recente Programa se situa, utilizando então, como dito, os indicadores de outro PPGCI de uma instituição federal, como base de comparação.

Como resultado complementar, este artigo contribui para o registro histórico da atuação do PPGCI UFSCar, ao registrar qual era a situação da produção científica dos seus docentes, antes desses atuarem em um PPGCl, almejando futuramente, ao replicar esta pesquisa, poder se instrumentalizar uma avaliação das orientações e estratégias implementadas, que demonstre o impacto positivo no aumento quantitativo e qualitativo da produção científica desses docentes.

$\mathrm{O}$ desenvolvimento de pesquisas desta natureza tem sido frequente e crescente na $\mathrm{Cl}$ nacional e internacional. Ao mesmo tempo uma análise crítica sobre as limitações dessas mensurações deve estar presente nesses estudos, para que tais instrumentos possam sempre ser aperfeiçoados e que o impacto social das pesquisas desenvolvidas também possa ser ponderado (HICKS et al., 2015). Esses indicadores são decisivos tanto para dar coerência e visibilidade ao que se tem produzido cientificamente em uma área do saber, tornado mais viável o desenvolvimento de uma análise crítica sobre este fazer, como se torna

\footnotetext{
1 "A área de Comunicação e Informação (31) é constituída pelas seguintes áreas básicas: Comunicação, Ciência da Informação e Museologia, com programas de Pós-Graduação em Arquivologia, Biblioteconomia, Ciência da Informação, Comunicação Jornalismo e Museologia. São as mesmas áreas básicas anteriormente reunidas nas Ciências Sociais Aplicadas I. Em 2016, a CAPES aprovou a alteração do nome, reconhecendo que a designação pregressa uma vez que não havia área de Ciências Sociais Aplicadas 2 - era imprecisa e vinha sendo responsável por enganos frequentes no encaminhamento pela comunidade de APCNs e pedidos de auxílio de modo geral, acarretando atrasos e, eventualmente, prejuízo aos interessados." (COORDENAÇÃO..., 2017, p. 1).
} 
estratégico enquanto indicador para o fomento e o financiamento de pesquisas futuras a serem desenvolvidas por este campo, seja pelo setor público ou privado.

O Journal of Informetrics e o Cybermetrics configuram-se atualmente como principais fontes de publicação dos resultados de pesquisas sobre as diferentes metrias, que têm sido desenvolvidas e aplicadas para diferentes finalidades. O periódico Knowledge Organization, assim como os eventos ISKO (Information Society for Knowledge Organization), específicos da $\mathrm{Cl}$, também acomodam investigações sobre o tema. No Brasil, o Grupo de Trabalho "Produção e Comunicação da Informação em Ciência, Tecnologia \& Inovação" (GT 7) do ENANCIB (Encontro Nacional de Pesquisa em Ciência da Informação) concentra pesquisas desenvolvidas e em desenvolvimento sobre $\mathrm{O}$ assunto. Soma-se a este o evento "Encontro Brasileiro de Bibliometria e Cientometria" EBBC, cujo objetivo assemelha-se ao GT indicado, mas que também aglutina trabalhos desvinculados de Programas de Pós-Graduação. Os objetivos dos trabalhos desenvolvidos são variados, e neste conjunto, situamos a proposta do presente artigo, que visa analisar o comportamento e a vocação da produção científica do corpo docente de um PPGCI recém-criado, utilizando como parâmetro, dados produzidos por docentes vinculados a um programa de excelência na área.

Sobre a criação do PPGCI UFSCar, ao longo dos 20 anos de existência do Departamento de Ciência da Informação (DCl) da UFSCar, houve interesse em se estabelecer um corpo docente que pudesse sustentar a proposta de criação de um PPG CI na UFSCar. Esse interesse foi fortalecido pelas iniciativas de melhoria de infraestrutura tanto de recursos humanos como de equipamentos e materiais, ocorridas muito em detrimento da participação do $\mathrm{DCl}$ no Programa REUNI (Programa de Apoio a Planos de Reestruturação e Expansão das Universidades Federais). Assim, em 2014, depois de aproximadamente 10 anos de movimentos e ações direcionadas à constituição de um ambiente favorável para a criação de uma pós-graduação em $\mathrm{Cl}$, foi feita uma avaliação interna, entre os docentes desse departamento, interessados na construção de um PPG, e assim, foi criada a comissão de elaboração da proposta, pensada coletivamente seguindo interesses globais, regionais e locais. Segundo o 
documento produzido para sua criação "[...] o objetivo do PPGCI UFSCar é fortalecer o campo da $\mathrm{Cl}$ enquanto área de produção de conhecimentos e métodos para a inovação, considerando sua perspectiva social e integralizadora" (UNIVERSIDADE..., 2017).

Conhecimento, Tecnologia e Inovação é a área de concentração do PPGCI UFSCar, tornando-se o primeiro programa da área que contempla a inovação na sua atuação como estratégia para a construção e difusão do conhecimento científico e tecnológico. Na UFSCar, este também é o único dos 50 PPGcs existentes, que indica a inovação enquanto área de concentração (SILVA; GRACIOSO, 2018). A delimitação desta área para o PPGCI procura respeitar concomitantemente os eixos de pesquisa em Ciência da Informação, as características de pesquisa do corpo docente que compõe seu quadro permanente, as características institucionais da UFSCar e regionais da cidade de São Carlos, reconhecida como polo tecnológico e de inovação (UNIVERSIDADE... 2017).

A área de concentração delimitada confere ao PPGCl UFSCar a responsabilidade de identificar e construir conceitos, métodos, teorias e ações que concatenem a ciência, a informação, a inovação e a sociedade. Assim, avaliar a produção cientifica dos docentes permanentes desse programa, nesse seu momento embrionário, pode ser decisivo para a construção de estratégias que fortaleçam sua vocação na produção do conhecimento sobre esses assuntos.

\section{CAPES E OS CRITÉRIOS DE AVALIAÇÃO DOS PROGRAMAS DE PÓS- GRADUAÇÃO}

A fim de manter uma boa qualidade nos cursos de mestrado e doutorado no Brasil, a CAPES é responsável pela avaliação do Sistema Nacional de PósGraduação (SNPG), atividade fundamental que foi estabelecida a partir de 1998. Para tanto, a CAPES identifica como objetos de avaliação a certificação de qualidade da pós-graduação brasileira, que se torna referência para distribuição de recursos e bolsas à pesquisa, além de identificar no SNPG se há desequilíbrio e potenciais áreas para a criação de novos programas no país. 
(COORDENAÇÃO..., 2015).

Os processos de avaliação compreendidos pelo SNPG podem ser divididos ao que se referem à entrada e permanência dos cursos de mestrado profissional, acadêmico e doutorado. Há uma avaliação inicial de uma proposta de novo curso de pós-graduação e a avaliação periódica dos cursos já existentes, prevendo a qualidade desses cursos. Quando um curso é aprovado pelo MEC, ele passa a fazer parte do SNPG e será avaliado de acordo com as condições estipuladas pela CAPES (COORDENAÇÃO..., 2015).

Para tanto, os processos de avaliação são sempre baseados no reconhecimento e a confiabilidade analisada por pares com critérios debatidos e atualizados pela comunidade acadêmica a cada período. Todo o processo envolve transparência, desde a tomada de decisões até a divulgação dos resultados pelo portal da CAPES (COORDENAÇÃO..., 2015).

A CAPES estabelece 49 áreas de avaliação, para as quais há disponíveis ofícios, comunicados, relatórios, apresentações e documentos gerais específicos. Um dos documentos mais relevantes para o processo de avaliação é o Documento de Área, que contém considerações sobre o estado atual de cada uma das áreas, sobre os critérios utilizados para avaliação e todas as características e perspectivas pertinentes, contemplando as diretrizes, para que um PPG possa se estabelecer, se identificar e para que possa buscar os norteamentos de sua existência. A CAPES disponibiliza, ainda, em seu portal outros dois documentos para os PPGs: as fichas de avaliação e os relatórios de avaliação, nos quais estão detalhados e apreciados especificamente os itens de avaliação e os critérios utilizados nas análises individualmente, avaliação esta, que passou a ser feita quadrienalmente em 2013.

Em cada Ficha de Avaliação estão descritos os cinco itens avaliados (Proposta do Programa; Corpo Docente; Corpo Discente, Teses e Dissertações; Produção Intelectual; Inserção Social) e seus respectivos pesos. Os indicadores a respeito da produção científica qualificada são analisados nos itens Produção Intelectual, Corpo Docente e Discente, Teses e Dissertações, e esses 3 itens juntos têm peso que pode variar entre $80 \%$ a $90 \%$ na avaliação dos programas. (BASSOLI, 2017). 
Essas informações são coletadas atualmente pela CAPES por meio da Plataforma Sucupira, que contempla todas as informações pertinentes sobre os PPGs. A Plataforma Sucupira é dividida em vários módulos e seu preenchimento é de responsabilidade individual de cada programa. Por ser uma plataforma online, os dados são alimentados (preferencialmente) à medida que surgem durante o ano, havendo uma data limite, para o envio e homologação dessas informações anualmente, que ficam disponíveis para consultas públicas a partir do momento em que os módulos forem preenchidos pelos PPGs.

Em consulta realizada na Plataforma Sucupira foi possível identificar 15 programas acadêmicos ( $15 \mathrm{com}$ mestrado e $11 \mathrm{com}$ mestrado e doutorado) que pertencem a área de Ciência da Informação e que estavam ativos em $2017^{2}$. Esses números indicam um crescimento relevante frente aos dados apontados na pesquisa de Gomes (2009), na qual existiam apenas 10 PPGs dessa área de concentração no país e dos quais somente metade possuíam o nível de doutorado.

As 49 áreas de avaliação estabelecidas são resultado da distribuição feita a partir de um total de nove grandes áreas, separadas dentre as de maior concentração até as de um nível mais específico de conhecimento. (COORDENAÇÃO..., 2016). Dentre essas áreas a Cl, com características interdisciplinares, está incluída nas CSAI (Comunicação e Informação - 2017).

Analisando pontualmente os critérios utilizados nas avaliações de doutorado e mestrado acadêmico em CSAI, entende-se que a nota é atribuída conforme cinco itens: Proposta do Programa; Corpo Docente; Corpo Discente, Teses e Dissertações; Produção Intelectual; Inserção Social. Para cada requisito, segundo o documento de área, é atribuído um conceito (insuficiente, fraco, regular, bom e muito bom) e cada item possui um peso específico na nota final. A Proposta do Programa é considerada o ponto inicial para avaliação geral do programa, mas não tem peso na avaliação, os indicadores referentes ao Corpo Docente têm peso de 20\%, Corpo Discente, Teses e Dissertações tem peso de $30 \%$, a Produção Intelectual tem peso de $40 \%$ e a Inserção Social e

\footnotetext{
${ }^{2}$ Em 2018 novos programas de mestrados foram aprovados um novo doutorado.
} 
Relevância tem peso $10 \%$, de modo que, os itens que envolvem a produção científica totalizam $90 \%$ da nota de um programa da área de CSAI.

A avaliação é dividida em pelo menos duas etapas, na qual, primeiramente todos os programas são avaliados e são atribuídas notas de 3 (três) a 5 (cinco). Na segunda etapa examina-se a possibilidade de atribuir nota 6 (seis) ou 7 (sete) aos programas que foram pontuados anteriormente com nota 5, caso atendam aos critérios de desempenho, solidariedade e nucleação, e inserção internacional (COORDENAÇÃO..., 2013e).

O Documento de Área das CSAI atribui valor à produção de artigos em periódicos, livros, produção artística e produção técnica, não pontuando trabalhos que foram apenas apresentados em eventos científicos. Sendo, a produção intelectual, pontuada a partir de critérios do Qualis-Periódicos, da Classificação de Livros e da Classificação da Produção Técnica (instrumentos específicos da CAPES), de forma que possam ser avaliados qualitativa e quantitativamente.

O uso do Qualis-Periódicos para a avaliação da qualificação das publicações em artigos pelos docentes permanentes vinculados a PPGs é feito por um comitê especializado da CAPES. A lista de classificação é feita a posteriori, a partir das informações enviadas anualmente pelos PPGs por meio da Plataforma Sucupira. Os periódicos que são listados nas publicações de docentes permanentes de PPGs são avaliados e distribuídos entre os estratos A1, A2, B1, B2, B3, B4, B5 ou C (BARATA, 2016). Sendo assim, espera-se que um pesquisador de alto desempenho tenha publicações em periódicos qualificados nos estratos mais altos da classificação.

Para avaliação de livros alguns critérios mínimos são pré-estabelecidos. No que diz respeito à categorização de uma publicação científica como livro é preciso: possuir um ISBN ou ISSN; mínimo de 50 páginas; ficha catalográfica idêntica à obra; e participação de docente e discente vinculado ao programa de pós-graduação. É importante destacar que a área de ciências humanas e sociais possui ações diferentes quanto ao principal meio de publicação,

[...] nas ciências exatas e naturais a tendência é que os resultados de pesquisa sejam expostos na forma de artigos pois a linguagem cifrada permite uma posição concisa e clara do que foi realizado. Já nas ciências sociais e humanas, a tendência é que se publique os resultados de pesquisa em livros, pois 
geralmente necessita-se de apresentação textual extensa para dar conta da complexidade das análises feitas. (SILVA; MENEZES; PINHEIRO, 2003, p. 194).

A avaliação da produção bibliográfica de livros é complexa e envolve critérios particulares para área. Cada critério contempla quesitos a serem avaliados de forma mais específica, quanto a seus aspectos formais e de forma qualitativa do conteúdo da obra, a fim de identificar sua relevância para a área.

\section{APRESENTAÇÃO E DISCUSSÃO DOS RESULTADOS}

As análises e as inferências foram feitas por meio da comparação do desempenho do PPGCI UFSCar em relação ao PPGCI UFMG, escolhido por alcançar nota 6 na avaliação, ser vinculado a uma Instituição Federal e ter mais de 40 anos de experiência. A UFMG, em 2016 passou a ofertar dois PPG, após a criação do PPG em Gestão \& Organização do Conhecimento, mas como esta pesquisa foi focada na última avaliação trienal - na qual havia um único PPG na UFMG, o chamado Ciências da Informação - a análise foi feita considerando esse único PPG.

Tendo como destaque desta pesquisa o campo da Ciência da Informação e a produção científica proveniente deste, analisou-se em particular os trechos que tratam sobre o que é avaliado e como é avaliado, com destaque à avaliação da produção científica, considerando suas medidas e pesos. Entretanto, para entender alguns conceitos específicos, principalmente quanto a produção científica, fez-se necessário analisar o Relatório de Avaliação 2010-2012: trienal 2013 (COORDENAÇÃO..., 2013e) bem como a Ficha de Avaliação individual dos programas já avaliados.

Após a análise documental, realizou-se em junho 2016, o levantamento da produção científica do período de 2013 a 2016 de todos os docentes vinculados ao DCI UFSCar, e posteriormente apenas dos docentes ativos no PPGCI UFSCar no período de 2016-2017, para então ser feita uma análise perante o documento de área das CSAI que regia a avaliação da CAPES.

O DCl apresenta um total de 16 docentes vinculados ao departamento, deste total, 10 estão vinculados como docentes permanentes do PPGCI UFSCar, 
ou seja, a maior parte do quadro docente possui ligação com o programa. $\mathrm{O}$ levantamento de dados contou com o processo de busca dos Currículos Lattes dos professores, que compõem atualmente o PPGCI UFSCar, a identificação e a recuperação dos dados referenciais sobre produção científica. As informações foram registradas e tratadas no programa Microsoft Excel, a fim de melhor analisar e visualizar os indicadores através de gráficos e tabelas.

Desse modo, seguindo as diretrizes do documento de área e outros documentos pertinentes elaborados pela CAPES, os itens possíveis de reprodução foram analisados para o PPGCI UFSCar, assim como os dados do PPGCI UFMG. Para realizar a análise quantitativa comparativa dos dois programas, foi estudada a Ficha de Avaliação do PPG UFMG, divulgada ao final da avaliação, na qual se encontram informações relevantes sobre a atribuição do conceito final, além de detalhes sobre a atribuição dos conceitos ao programa. (COORDENAÇÃO..., 2013e).

\subsection{PONTUAÇÃO DO PPGCI UFSCAR SEGUINDO DOCUMENTO DE ÁREA EM CSAI (COMUNICAÇÃO E INFORMAÇÃO - 2017)}

Nas Ciências Sociais Aplicadas I/Comunicação e Informação, que abrange a atuação dos PPGCl, a avaliação da produção científica, compreende a atuação do PPG como um todo, ao analisar a produtividade individual e a sua distribuição entre os docentes. Para tanto, atribui pesos às publicações de acordo com o estrato Qualis-Periódicos. Nessa avaliação se um artigo é publicado em conjunto por dois docentes do programa, este é contado somente uma vez na avaliação do programa. O indicador apresentado no Gráfico 1 externaliza a distribuição da produção científica do PPGCI UFSCar, em relação ao estrato Qualis-Periódicos, indicando a pontuação obtida no período analisado entre 2013-2016. Foram publicados 42 artigos pelos docentes do PPGCI UFSCar, destes, $95 \%$ foram em periódicos classificados nos estratos mais altos do Qualis-Periódicos (A1 a B2). A pontuação obtida é utilizada pela comissão de avaliação que, juntamente com outros indicadores, atribui o conceito final sobre o desempenho do PPG, na categoria produção intelectual. No caso, o PPGCl UFSCar apresentou a maior parte da sua pontuação vinda principalmente do 
estrato Qualis-Periódicos B1 seguido de A1, sinalizando a qualidade da sua produção científica. Porém, aponta a necessidade de avançar em estratégias que possam impactar na internacionalização de suas contribuições para a área. Como por exemplo, investir em iniciativas de redes de colaboração, com a participação de pesquisadores internacionais, o que pode contribuir para impulsionar o impacto da produção, ao criar conexões, compartilhar responsabilidades e competências, otimizar a utilização de recursos, aumentar a visibilidade das publicações entre outros benefícios (FUNARO et al., 2009).

\section{Gráfico 1- Distribuição das publicações dos docentes do PPGCI UFSCar por estrato Qualis-Periódicos}

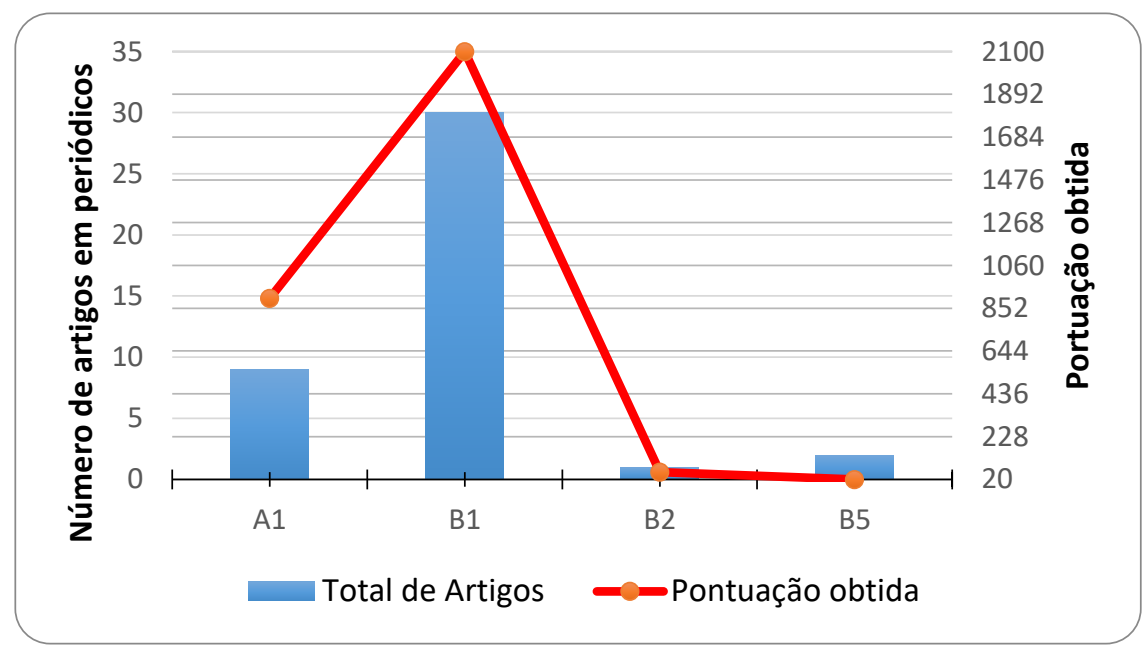

Fonte: Elaborado pelos autores.

Visando entender o comportamento de publicação dos docentes do PPGCI UFSCar, foi elaborado o Gráfico 2, que apresenta a distribuição dos 42 artigos publicados no período de 2013-2016, por ano e por docente. Optou-se neste artigo por omitir os nomes desses docentes, que foram representados por letras (A; B; C; D; E: F; G; H; I; J) nos Gráficos 2 e 3. Apesar do PPGCI UFSCar manter um padrão quanto a quantidade de publicações por ano no período analisado, foi possível observar que há docentes com mais de duas publicações em um determinado ano e nenhuma em outro ano, assim como docentes que nem mesmo apresentam publicações em nenhum dos anos. Este indicador se torna importante, não somente frente a avaliação da CAPES, mas também para as boas práticas de gestão do PPG, ao subsidiar a elaboração, manutenção e 
aplicação de regulamentos internos sobre credenciamento e recredenciamento de docentes em um PPG.

Baseando-se no documento de área das CSAI do quadriênio 2013-2016, foi atribuído valores para os 42 artigos publicados em periódicos de acordo com o seu estrato Qualis-Periódicos na área. O PPGCI UFSCar alcançou um total de 3.555 pontos, uma média de 355,5 pontos por docente. O Gráfico 3 apresenta os valores alcançados individualmente pelos docentes do PPGCI UFSCar, foi possível visualizar que 06 docentes apresentam valores acima e 02 docentes apresentam valores significativamente abaixo da média do PPGCI UFSCar. Deve-se atentar que o docente, que não apresenta publicações qualificadas na área, abaixa a média dos outros docentes, contribuindo para a avaliação negativa do programa, quanto ao número e qualidade das publicações.

Gráfico 2 - Distribuição das publicações por docentes e ano

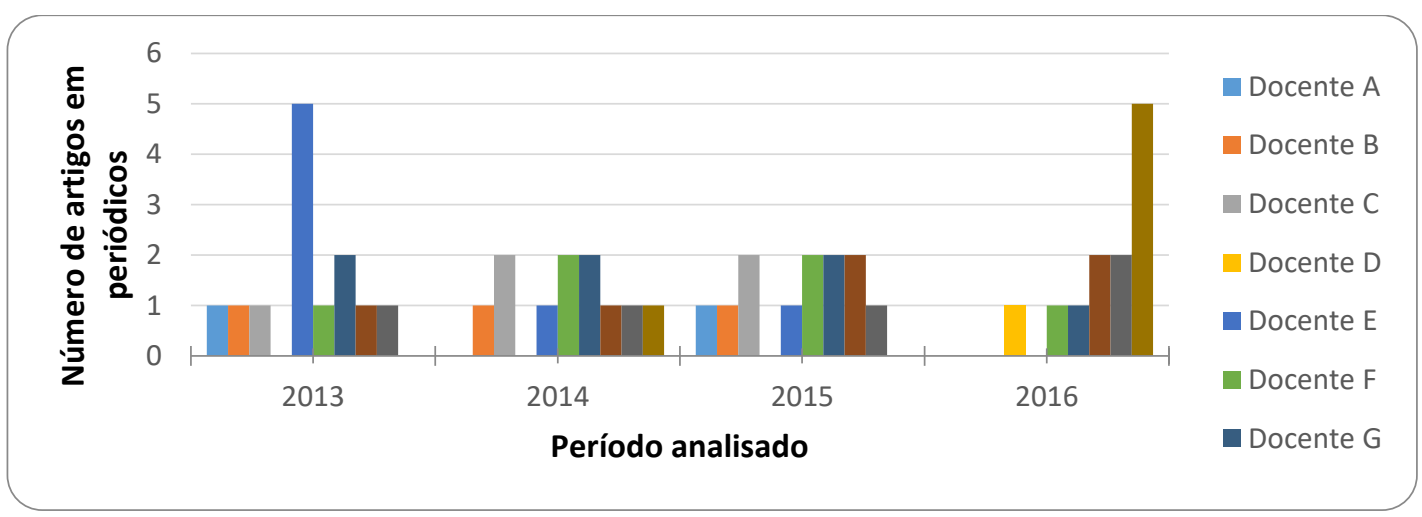

Fonte: Elaborado pelos autores.

Gráfico 3 - Soma dos pesos, com base no Qualis-Periódicos, atribuídos às publicados dos docentes do PPGCI UFSCar

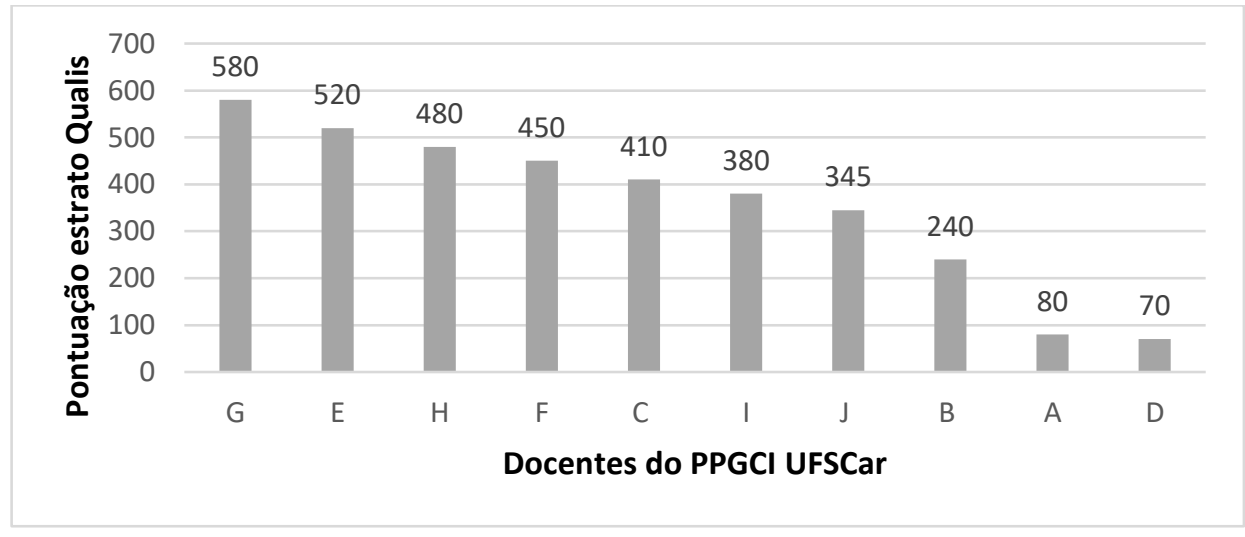

Fonte: Elaborado pelos autores. 
Além disso, a avaliação também é feita ao nível de docente permanente (indivíduo). A análise quantitativa das publicações através das coautorias entre docentes, permite identificar que há parcerias internas ao mesmo tempo que há publicações individuais no programa. O Gráfico 4 apresenta a média individual alcançada pelos docentes do PPGCI UFSCar no período analisado (2013-2016), essa média foi construída a partir da soma dos valores atribuídos aos artigos publicados pelo docente, de acordo com o estrato Qualis-Periódicos, dividida pelo número total de artigos publicados pelo docente. A análise qualitativa da produção intelectual fica evidente neste momento, ressaltando a importância de se atentar a qualidade das publicações e não somente a quantidade, motivo de muitas discussões, uma vez que esta tende a evitar o chamado produtivismo quantitativo (COORDENAÇÃO, 2013b; KUHLMANN JR., 2015), mas que, ainda assim, aparece como critérios para geração de conceitos finais no sistema de avaliação da CAPES.

\section{Gráfico 4 - Média individual alcançada no período analisado}

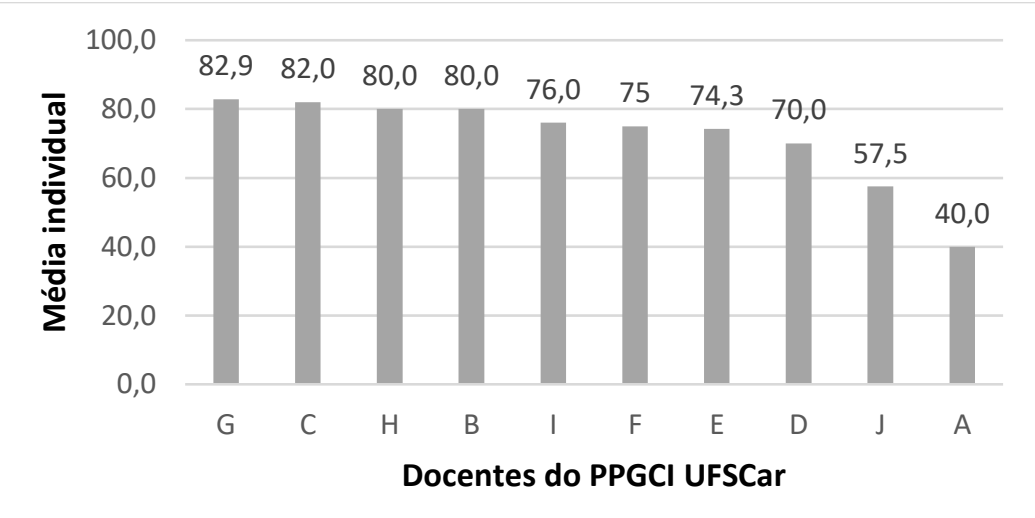

Fonte: Elaborado pelos autores.

Com isso é possível notar que os docentes mantêm um padrão considerado alto frente ao quantitativo de publicações que possuem, com uma média de 77,77 pontos. Deve-se atentar, entretanto, àqueles que não atingem esse número, já que a avaliação é feita sobre o programa como um todo, contribuem para a avaliação negativa do programa.

A produção intelectual envolvendo a publicação de livros, capítulos de livros e trabalhos em eventos, passa pela avaliação qualitativa de um comitê específico da área. Essa tipologia de produção intelectual é valorizada pela 
comunidade científica da área de Ciência da Informação, principalmente por envolver uma troca de experiências na área com outros pesquisadores, o que conta muito para avaliação de um programa. Devido à dificuldade em se atribuir as pontuações aos capítulos de livros e livros publicados, não foi possível realizar a valoração dessa produção intelectual. Quanto aos trabalhos completos publicados em eventos científicos, foi possível gerar uma estimativa, com base na Tabela de Classificação de Livros (COORDENAÇÃO..., 2013a), que apresenta a classificação atribuída aos anais de eventos da área. O Gráfico 5 , apresenta a distribuição de 109 trabalhos publicados em eventos científicos, pelos docentes do PPGCI UFSCar, por estrato na Classificação de Livros, no período de 2013-2016.

\section{Gráfico 5 - Distribuição dos trabalhos publicados em anais de eventos por estrato na Classificação de Livros}

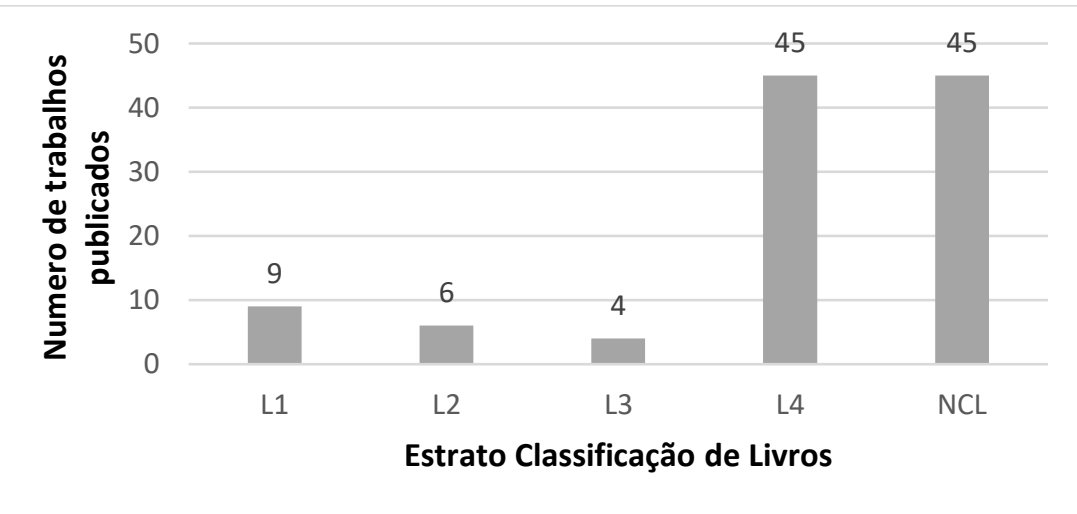

Fonte: Elaborado pelos autores

O estrato NCL comporta os trabalhos que não foram incluídos na Classificação de Livros, e por isso não foram pontuados. Nesta dimensão da avaliação, após valorar os trabalhos publicados, o PPGCI UFSCar alcançou 4.875 pontos, no período analisado. Nota-se o significativo volume de publicações no estrato $L 4$, que apresenta o maior peso atribuído pela CAPES aos Anais de eventos científicos, o que, além de indicar a qualidade das publicações, demonstra que os docentes do PPGCI UFSCar buscam participar de eventos científicos, reconhecidos pela comunidade da Ciência da Informação, como locais legítimos para o compartilhamento de experiências e de conhecimentos na área. 


\subsection{O PPGCI UFSCAR COMPARADO AO PPGCI UFMG}

Comparou-se o desempenho do PPGCI UFSCar com o PPGCI UFMG, visando subsidiar a elaboração de uma política de produção científica para o PPGCI UFSCar. Essa comparação focou o resultado da avaliação da produção intelectual dos docentes do PPGCI UFSCar, em especial a ponderação da produção em periódicos científicos e em eventos, além de informações relevantes sobre o quadro docente e relevância das publicações. As análises da Ficha de Avalição do PPGCI UFMG, disponível na Plataforma Sucupira, tiveram como foco o item 4, Produção Intelectual, apresentados no Quadro 1.

\section{Quadro 1 - Quadro de avaliação da Produção Intelectual da UFMG}

\begin{tabular}{|c|c|c|}
\hline \multicolumn{3}{|l|}{4 - PRODUÇÃO INTELECTUAL } \\
\hline Itens de Avaliação & Peso & Avaliação \\
\hline 4.1. Publicações qualificadas do Programa por docente permanente. & 40.00 & Muito Bom \\
\hline $\begin{array}{l}\text { 4.2. Distribuição de publicações qualificadas em relação ao corpo docente } \\
\text { permanente do Programa. }\end{array}$ & 30.00 & Muito Bom \\
\hline 4.3. Produção técnica, patentes e outras produções consideradas relevantes. & 15.00 & Bom \\
\hline 4.4. Produção artística, nas áreas em que tal tipo de produção for pertinente. & 15.00 & Muito Bom \\
\hline & Comissão & Muito Bom \\
\hline
\end{tabular}

Fonte: Ficha de Avaliação UFMG 2013. (COORDENAÇÃO, 2013c).

Diante do Quadro 2, nota-se que esta pesquisa conseguiria analisar os itens "4.1 Publicações qualificadas do Programa por docente permanente" e "4.2 Distribuição de publicações qualificadas em relação ao corpo docente permanente do Programa", entretanto, diante de uma tentativa de reproduzir os cálculos acerca de tais atribuições, encontraram-se falhas envolvendo o acesso ao conhecimento, sobre alguns pontos e índices contabilizados para se chegar a média final, além de que, algumas informações estariam ausentes na avaliação do PPGCI UFSCar, em especial os valores pertinentes aos livros e capítulos de livros publicados. Dessa forma, analisou-se as médias finais obtidas pelo PPGCI UFMG nos dois itens (4.1 e 4.2), comparando-as com o desempenho alcançado pelo PPGCI UFSCar.

No item "4.1 - Publicações qualificadas do Programa por docente permanente" - o PPGCI UFMG obteve uma média final de 124,1 pontos, 
atribuindo a ele o conceito de "Muito Bom". Para tanto foram considerados os pontos em artigos, livros e capítulos. A Figura 1 apresenta a Ficha de Avaliação do PPGCI UFMG, com a distribuição de pesos para as publicações qualificadas.

\section{Figura 2 - Distribuição de pesos para as Publicações Qualificadas do PPGCI} UFMG

O PPG obteve, nesse processo, a seguinte pontuação:
a) Pontos em artigos - $11.015,0$
b) Pontos em livros, capitulos e complementares - 11.207,50
c) Total de pontos brutos ("a" + "b") - 22.222,5
d) Número total de itens produzidos - 302
e) Média anual de pontos ("c"/3) - 7.407,5

f) Média anual de docentes - 21,3

g) Média anual de itens produzidos ("d"/3) - 101

h) Média de pontos por docente ("e"/"f") - 347,8

i) Indice $1-1,6$

j) Média de pontos por produto ("e"/"g") - 73,6

k) Indice $2-1,2$

1) Média Final $-124,1$

Fonte: Ficha de Avaliação UFMG 2013. (COORDENAÇÃO, 2013c).

Após a análise da Ficha de Avaliação (Figura 2) foi possível intuir que os itens "a", "b", "c" e "d" eram possíveis de serem identificados e valorados para o PPGCI UFSCar, onde "b" e "c" seriam, neste caso, somente referentes as pontuações em eventos. Assim, diante dos resultados apresentados na subseção anterior o desempenho do PPGCI UFSCar no período analisado é apresentado no Quadro 2.

\section{Quadro 2 - Pontuação atribuída ao PPGCI UFSCar no item "4.1" da Ficha de Avaliação 2013}

\begin{tabular}{|l|l|}
\hline Item & Pontuação PPGCI \\
\hline Pontos em artigos & 3.555 \\
\hline Pontos em capítulos (eventos) & 4.875 \\
\hline Total de pontos brutos (artigos + eventos) & 8.430 \\
\hline Número total de itens produzidos & 259 \\
\hline
\end{tabular}

Fonte: Elaborado pelos autores.

No processo de identificação e valoração da produção intelectual do PPGCI UFSCar, optou-se por não adicionar os itens da Ficha de Avaliação (Figura 2), que exigiam uma ponderação de médias, considerou-se somente os 
valores brutos, ou seja, a soma das produções que conseguiram ser contabilizadas pela pontuação simples (artigos e eventos). O PPGCI UFSCar apresentou um total de 259 itens para 10 docentes permanentes num período de quatro anos (2013-2016). Já o PPGCI UFMG, em um período de três anos, com 22 docentes permanentes, obteve um total de 302 itens publicados.

Diante destes resultados, pode-se afirmar que o PPGCI UFSCar apresenta desempenho na produção intelectual e um quadro de docentes, compatíveis com a atribuição do conceito "bom", diante da avaliação da CAPES, pois, essa produção é relevante para área e está distribuída entre o corpo docente.

Quanto ao item "4.2 Distribuição de publicações qualificadas em relação ao corpo docente permanente do Programa". Seguindo as diretrizes de cálculo desse item, foram identificadas as seis produções de maior pontuação de cada docente, então, dividiu-se a pontuação dessas publicações por 6, para obter a média de pontos por docente, o resultado foi somado ao PPGCI UFSCar e então dividido pelo número total de docentes permanentes. Entretanto, por esse item considerar também, questões como a permanência de docentes no programa e pontuações às publicações que não foram possíveis de se realizar neste artigo, por se tratarem essencialmente de aspectos qualitativos definidos pelo comitê da área, não foi possível aplicar integralmente as considerações do item "4.2" ao PPGCI UFSCar. Porém, o indicador apresentado no Gráfico 4, apresenta uma valoração considerável alcançada pelos docentes do PPGCI UFSCar. O PPGCI UFMG obteve no item "4.2" na última avaliação o conceito de "muito bom", atribuído a partir da média de 84,5 pontos por docente permanente.

Como a Ficha de Avaliação da CAPES possui muitas questões complexas, que abriram margens para dúvidas e que não foram sanadas em nenhum outro documento da CAPES da área de CSAI, uma análise menos complexa pôde ser realizada a partir da Planilha de Indicadores, disponível no site da CAPES da última avaliação trienal, que contém dados brutos de todos os programas que foram avaliados (COORDENAÇÃO..., 2013d). Analisando os dados somente do PPGCI UFMG, com 22 docentes na ocasião desta pesquisa, tem-se o quantitativo de artigos em periódicos (considerando o estrato Qualis- 
Periódicos A1 à B5) de 196 publicações em três anos, enquanto o PPGCl UFSCar, no período analisado, com 10 docentes, obteve um quantitativo de 42 publicações. Neste item é interessante ressaltar que, apesar da diferença significativa na quantidade de publicações, ambos os programas concentram suas publicações em periódicos A1 e B1, considerados os mais relevantes para área de $\mathrm{Cl}$, o que sugere um comportamento da área.

Além disso, o número de trabalhos publicados em eventos pelos docentes do PPGCI UFMG é superior ao de artigos em periódicos, tal situação se assemelha ao PPGCI UFSCar, onde há um total de 64 trabalhos publicados em eventos classificados no estrato Qualis-Periódicos da área. Quanto a publicação de livros, o PPGCI UFSCar possui um total de 12 livros e 50 capítulos de livros publicados no período analisado, e mais uma vez, apesar da diferença entre os quantitativos dos programas, o PPGCI UFSCar já apresenta docentes participantes de questões da área e que, agora inseridos no sistema de pósgraduação nacional, tendem a crescer e ajudar o campo da $\mathrm{Cl}$.

Assim, embora se evidencie que quantitativamente a produção científica dos docentes permanentes do PPGCI UFSCar, ainda, se mostra incipiente em relação ao mesmo quantitativo de um programa nota 06 , visualiza-se uma aderência do comportamento de produção intelectual do PPGCI UFSCar com o comportamento da área, reforçado pela vocação do programa para desenvolver publicações qualificadas.

\section{CONSIDERAÇÕES FINAIS}

A partir da criação do PPGCI UFSCar no ano de 2016 e da necessidade de se compreender os critérios e mecanismos que regem a avaliação da pósgraduação brasileira, este artigo teve como objetivo elaborar e aplicar uma sistemática para o diagnóstico do potencial de atuação de um PPG da área de Ciência da Informação, sob a perspectiva da avaliação da CAPES, utilizando o PPGCI UFMG nota 06, como base de comparação quantitativa e qualitativa, para subsidiar a discussão de alguns elementos previstos no documento de área de Ciências Sociais Aplicadas (Comunicação e Informação - 2017). 
No que diz respeito à produção intelectual dos docentes permanentes do PPGCI UFSCar, é possível constatar o engajamento e a pró-atividade dos mesmos na submissão dos resultados de pesquisas para publicação em eventos e revistas científicas qualificadas na área. Este movimento presente nas atividades de pesquisa dos docentes, já se configura como resultado de um planejamento a longo prazo para a proposição e a implementação de um PPGCl na UFSCar. Dados complementares de participação como pareceristas em eventos e revistas do campo, também poderiam ser analisados futuramente para confirmar este momento de engajamento com a área.

Contudo, quando comparado a um programa nota 06, evidenciam-se suas fragilidades, demandando a configuração de estratégias que precisarão ser articuladas e estruturadas, visando otimizar tanto a construção de redes de colaboração entre os pesquisadores do PPGCI UFSCar, como também entre os pares vinculados a outras instituições nacionais e internacionais.

Quanto a análise complementar feita sobre o documento de área da CAPES (Ciências Sociais Aplicadas I/Comunicação e Informação), pode-se considerar que a CAPES, mesmo possuindo alguns critérios já consolidados, no que diz respeito à avaliação da produção científica de um PPGCl, ainda poderia investir na melhoria da acessibilidade das informações presentes nos relatórios de avaliação, promovendo a articulação de dados e facilitando a realização de análises comparativas entre os programas, para que os mesmos tenham uma visualização, mais legítima e apropriada dos percursos de produção de pesquisa que estão assumindo, na busca pelo avanço do campo da Ciência da Informação. Esta sugestão se faz, tendo em vista que foi somente a partir da análise comparada entre a produção científica de um PPGCl iniciante com um PPGCI já consolidado, que se tornou possível identificar quais as lacunas e quais seriam as estratégias futuras para que um novo programa possa ser reconhecido na sua futura atuação, enquanto produtor científico no campo.

A sistemática utilizada neste artigo, compreendendo o levantamento das informações, bem como o detalhamento do documento que rege a avaliação da pós-graduação, e a análise comparativa de desempenho entre programas com diferentes estágios de evolução, pode auxiliar na gestão de um PPGCl, em 
especial para a compreensão do potencial de atuação existente dentro do quadro de docentes que compõe o programa. E a partir dessa compreensão elaborar estratégias visando mobilizar os atores deste processo (os docentes permanentes) a investirem em determinadas ações de produção científica, que possam contribuir para a promoção do impacto científico e social do PPG, enquanto produtor de conhecimento científico para a área de $\mathrm{Cl}$.

\section{REFERÊNCIAS}

ARAÚJO, C. A. A. Bibliometria: evolução histórica e questões atuais. Em Questão, Porto Alegre, v. 12, n. 1, p. 11-32, jan./jun. 2006. Disponível em: https://seer.ufrgs.br/EmQuestao/article/view/16/5. Acesso em: 01 jun. 2016.

ARAÚJO, C. A. A. O que é ciência da informação. Belo Horizonte: KMA, 2018.

BARATA, R. C. B. Dez coisas que você deveria saber sobre o Qualis. Revista Brasileira de Pós Graduação, v. 13, n. 30, p. 13-40, 2016. Disponível em: http://dx.doi.org/10.21713/2358-2332.2016.v13.947. Acesso em: 01 jun. 2016.

BARDIN, L. Análise de conteúdo. São Paulo: Edições 70, 2011.

BASSOLI, M. Avaliação do Currículo Lattes como fonte de informação para construção de indicadores: o caso da UFSCar. 2017. 128 f. Dissertação (Mestrado em Ciência, Tecnologia e Sociedade) - Universidade Federal de São Carlos, São Carlos, 2017.

BORKO, H. Information Science: What is it? American Documentation, v. 19, n. 1, p. 3-5, jan. 1968. Disponível em: https://doi.org/10.1002/asi.5090190103. Acesso em: 25 out. 2018.

COORDENAÇÃO DE APERFEIÇOAMENTO DE PESSOAL DE NÍVEL SUPERIOR (CAPES). Avaliação da pós-graduação: sobre avaliação.

Brasília, 2015. Disponível em: http://www.capes.gov.br/avaliacao/avaliacao-dapos-graduacao. Acesso em: 12 maio 2016.

Classificação de Livros. Brasília, 2013a. Disponível em: http://avaliacaotrienal2013.capes.gov.br/classificacao-de-livros. Acesso em: 03 jun. 2016.

Documento de área: avaliação trienal 2010-2012. Brasília, 2013b. Disponível em: http://www.avaliacaotrienal2013.capes.gov.br/documento-dearea-e-comissao. Acesso em: 03 jun. 2016. 
. Fichas de Avaliação. Brasília, 2013c. Disponível em:

http://avaliacaotrienal2013.capes.gov.br/resultados/fichas-de-avaliacao. Acesso em: 3 jun. 2016.

Planilhas de Indicadores: Ciências Sociais Aplicadas I. 2013d.

Disponível em: http://avaliacaotrienal2013.capes.gov.br/resultados/planilhascomparativas. Acesso em: 01 jun. 2016.

. Plataforma Sucupira. Brasília, 2016. Disponível em:

https://sucupira.capes.gov.br/sucupira/public/index.jsf. Acesso em: 03 jun. 2016.

Disponível em:

Relatório de Avaliação 2010-2012: Trienal 2013. Brasília, 2013 e.

http://www.avaliacaotrienal2013.capes.gov.br/relatorios-de-avaliacao. Acesso em: 03 jun. 2016.

Relatório da Avaliação Quadrienal 2017: Comunicação e Informação. Brasília, 2017. Disponível em:

http://www.capes.gov.br/component/content/article/44-avaliacao/4662-cienciassociais-aplicadas-i. Acesso em: 02 out. 2018.

FUNARO, V. M. B. O.; RAMOS, L. M. S. V. C.; CARDOSO, S. C.; MARICATO, J. de M. Redes sociais e sistemas de informação: o pesquisador da área da saúde. In: POBLACION, D. A.; MUGNAINI, R.; FUNARO, V. M. B. O. (Orgs.). Redes sociais e colaborativas em informação científica. São Paulo: Angellara, 2009. p. 347-373.

GIULIANI, A. A.; MARQUETTO, R. M. F.; TUSI, J. S.; BOFF, V., ÁVILA, L. V. Proposição de diretrizes estratégicas para elevar o conceito da pós-graduação: estudo de caso do PPGGEO. Revista Brasileira de Pós-Graduação, v. 13, n. 30, p. 225-249, 2016. Disponível em: http://dx.doi.org/10.21713/23582332.2016.v13.903. Acesso em: 25 out. 2018.

GOMES, M. Y. F. F. Desafios atuais da Ciência da Informação no Brasil. Perspectivas em Ciência da Informação, v. 14, n. 3, p. 190-205, 2009. Disponível em: http://www.scielo.br/scielo.php?script=sci_arttext\&pid=S141399362009000300012. Acesso em: 03 nov. 2016.

HICKS, D.; WOUTERS, P.; WALTMAN, L.; RIJCKE, S.; RAFOLS, Ismael. Bibliometrics: The Leiden Manifesto for research metrics. Nature, v. 520, n. 7548, p. 429-431, 2015. Disponível em: http://dx.doi.org/10.1038/520429a. Acesso em: 25 out. 2018.

KUHLMANN JR., M. Produtivismo acadêmico, publicação em periódicos e qualidade das pesquisas. Cadernos de Pesquisa, São Paulo, v. 45, n. 158, p. 838-855, dez. 2015. Disponível em: 
http://www.scielo.br/scielo.php?script=sci_arttext\&pid=S0100-

$15742015000400838 \&$ Ing=pt\&nrm=iso. Acesso em: 07 nov. 2018.

SILVA, D. G.; GRACIOSO, L. S. A pesquisa em Inovação na UFSCar: uma análise descritiva. In: SEMINÁRIO DE INFORMAÇÃO, INOVAÇÃO E SOCIEDADE, 2018. São Carlos. Anais... São Carlos: PPGCI, PPGCTS, 2018.

SILVA, E. L.; MENEZES, E. M.; PINHEIRO, L. V. Avaliação da produtividade científica dos pesquisadores nas áreas de ciências humanas e sociais aplicadas. Informação \& Sociedade, v. 13, n. 2, p. 193-222, 2003. Disponível em: http://www.periodicos.ufpb.br/ojs2/index.php/ies/article/view/97/1567.

Acesso em: 25 out. 2018.

UNIVERSIDADE FEDERAL DE SÃO CARLOS. Programa de Pós-Graduação em Ciência da Informação: sobre o programa. 2017. Disponível em: http://www.ppgci.ufscar.br/sobre. Acesso em: 03 mar. 2017.

\title{
DIAGNOSIS OF THE INTELLECTUAL PRODUCTION OF UFSCAR'S POST-GRADUATION IN INFORMATION SCIENCE AT THE MOMENT OF ITS IMPLEMENTATION: CHALLENGES AND POTENTIALITIES
}

\begin{abstract}
Introduction: The qualification, creation and expansion of Strictu Sensu Postgraduate Programs (PPG) are strategic and fundamental actions for Brazilian scientific and intellectual production. Consequently, these programs need to be constantly evaluated for improvement. In the field of Information Science $(\mathrm{Cl})$ these actions have intensified. Objective: To elaborate and apply a systematization for the diagnosis of the potential of a newly implemented PPGCl, in order to identify its behavior and vocation for the scientific production in the area. Methodology: Analysis of the content on the CAPES evaluation system's reports; identification and mapping of the permanent professors' scientific production on the Federal University of São Carlos' (UFSCar) PPGCl; establishment of comparative basis of the PPGCI UFSCar's intellectual production against Federal University of Minas Gerais' PPGCI. Results: Based on data analysis and established comparisons, it was possible to verify the engagement and proactivity of the UFSCar PPGCl's faculty in the submission of their research results for publication in events and qualified scientific journals in the area. At the same time, there are gaps in the collaboration between the researchers, both internal to the Program and linked to other institutions. Conclusions: The need to devise strategies to mobilize actors in this process to invest in scientific production and collaborative networks is important in order to contribute to the promotion of the scientific and social impact of the PPGCI. The methodology used has also shown to be effective when used by other PPGs in the early stages of implementation, which aim to establish strategies for their consolidation and growth.
\end{abstract}

Descriptors: Information Science. Scientific productivity. Information science schools. 


\title{
DIAGNÓSTICO DE LA PRODUCCIÓN INTELECTUAL DEL POSTGRADO EN CIENCIA DE LA INFORMACIÓN DE LA UFSCAR EN EL MOMENTO DE SU IMPLEMENTACIÓN: DESAFÍOS Y POTENCIALIDADES
}

\begin{abstract}
RESUMEN
Introducción: La calificación, creación y ampliación de Programas de Postgrado strictu sensu (PPG) son acciones estratégicas y fundamentales a la producción científica e intelectual brasileña. En consecuencia, estos programas deben evaluarse constantemente para mejorarlos. En el campo de la Ciencia de la Información (Cl) estas acciones se han intensificado. Objetivo: Elaborar y aplicar una sistemática para el diagnóstico del potencial de actuación de un PPGCI recién implementado, buscando identificar su comportamiento y vocación para la producción científica en el área. Metodología: Análisis de contenido de informes del sistema de evaluación de la CAPES; identificación y mapeo de la producción científica de docentes permanentes del PPGCl de la Universidad Federal de São Carlos (UFSCar); el establecimiento de base comparativa de la producción intelectual del PPGCI UFSCar, con el PPGCl de la Universidad Federal de Minas Gerais. Resultados: A partir del análisis de los datos y de las comparaciones establecidas, fue posible constatar el compromiso y la proactividad del cuerpo docente del PPGCI UFSCar en la sumisión de los resultados de sus investigaciones para publicación en eventos y revistas científicas calificadas en el área. Al mismo tiempo se evidencian lagunas en la colaboración entre los investigadores, tanto internos al Programa y vinculados a otras instituciones. Conclusiones: Se explicita la necesidad de elaborar estrategias para movilizar a los actores de este proceso a invertir en la producción científica y en redes de colaboración, de modo que puedan contribuir en la promoción del impacto científico y social del PPGCI. La metodología utilizada también se mostró eficaz para ser utilizada por otros PPGs en fases iniciales de implementación, que anhelan establecer estrategias para su consolidación y crecimiento.
\end{abstract}

Descriptores: Ciencia de la información. Productividad científica. Programa de Postgrado en Ciencia de la Información. 